\title{
Lattice Vibrations and Ideal Electrical Resistivity of Noble Metals
}

\author{
B. P. Singh and M. P. Hemkar \\ Department of Physics, University of Allahabad, Allahabad, India
}

Z. Naturforsch. 34a, 310-314 (1979); received November 15, 1978

\begin{abstract}
The phonon dispersion in the three principal symmetry directions $[\zeta 00],[\zeta \zeta 0]$ and $[\zeta \zeta \zeta]$ and the temperature variation of the electrical resistivity of $\mathrm{Cu}, \mathrm{Ag}$ and $\mathrm{Au}$ have been studied by using a lattice dynamical model which takes into account $d$ shell-d shell central interactions up to second neighbours. The calculated results have been compared with the available experimental data and have been found to be in a satisfactory agreement.
\end{abstract}

\section{Introduction}

A knowledge of the behaviour of the lattice waves is essential in the study of numerous physical properties of metals such as specific heat, thermal and electrical conductivity etc. The prolem of devising an adequate description of a lattice of ions subject to vibration remains a major one. Nevertheless, the lattice dynamics of metals has been the subject of a number of recent theoretical investigations.

Several phenomenological models $[1-5]$ have been proposed for the lattice vibrations of metals. These models essentially use the effect of the electron gas on the lattice vibrations explicitly on the basis of the de Launey [6], Bhatia [7], Sharma and Joshi [8], Krebs [9] and Cheveau [10] theories. However, all these lattice dynamical models suffer from one drawback or the other. In these case of the transition and noble metals the main drawback with these approaches is the neglect of the $d$ shell$d$ shell central interactions.

In the present paper we have used our model $[11,12]$, which takes into account the $d$ shelld shell central interactions up to the second neighbours for the calculation of dispersion curves and the temperature dependence of phonon limited electrical resistivities along the three principal

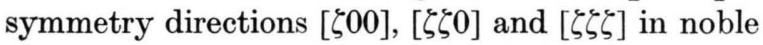
metals. This study is motivated by the fact that several authors have extensively investigated the elastic properties of the noble metals and that detailed experimental phonon dispersion curves for coherent inelastic neutron scattering and measurements of the temperature variation of the electrical resistivity over a wide range of temperature are available.

Reprint requests to B. P. Singh, 13-D/2 Muir Road, Rajapur Allahabad 211002, Indien.

$0340-4811 / 79 / 0300-0310 \$ 01.00 / 0$

\section{Theory}

The secular determinant determining the angular frequencies $\omega(=2 \pi v)$ of the normal modes of vibration in a cubic lattice can be written as

$$
\left|D(\boldsymbol{q})-M \omega^{2} I\right|=0,
$$

where $M$ is the mass of the atom, $\boldsymbol{q}$ the phonon wave vector and $I$ the unit matrix of order three. The diagonal and non-diagonal elements of the dynamical matrix $D(\boldsymbol{q})$ are:

$$
\begin{aligned}
D_{11}(\boldsymbol{q})= & -2 \alpha_{1}\left[2-C_{1}\left(C_{2}+C_{3}\right)\right] \\
& -4 \alpha_{2} S_{1}^{2}-K+K^{2} / N, \\
D_{12}(\boldsymbol{q})= & -2 \alpha_{1} S_{1} S_{2},
\end{aligned}
$$

where $C_{i}=\operatorname{Cos}\left(a q_{i}\right), S_{i}=\operatorname{Sin}\left(a q_{i}\right), i=1,2,3$, and $a$ is semilattice parameter. The value of $N$ can be evaluated from the determinant

$$
\left|D^{\prime}(\boldsymbol{q})-N I\right|=0 \text {. }
$$

The typical elements of the dynamical matrix $D^{\prime}(\boldsymbol{q})$ are expressed as

$$
\begin{aligned}
D_{11}^{\prime}(\boldsymbol{q})= & 2 \beta_{1}\left[2-C_{1}\left(C_{2}+C_{3}\right)\right] \\
& +4 \beta_{2} S_{1}{ }^{2}+K-A^{\prime} G(\boldsymbol{q}), \\
D_{12}^{\prime}(\boldsymbol{q})= & 2 \beta_{1} S_{1} S_{2} .
\end{aligned}
$$

Here we have denoted the force constants for ion core-ion core central interactions out to second neighbours by $\alpha_{1}$ and $\alpha_{2}$, nearest and next nearest neighbours d shell-d shell central interactions by $\beta_{1}$ and $\beta_{2}$, ion core-d shell interactions by an isotropic constant $K$ and d shell-conduction electrons interactions by $A^{\prime} . G(\boldsymbol{q})$ is expressed as

$$
\begin{aligned}
A^{\prime} G(\boldsymbol{q})= & A^{\prime} \sum_{\boldsymbol{H}}\left[\frac{(\boldsymbol{q}+\boldsymbol{H})(\boldsymbol{q}+\boldsymbol{H}) \cdot \boldsymbol{e}}{|\boldsymbol{q}+\boldsymbol{H}|^{2}+k_{\mathrm{c}}{ }^{2}} G^{2}\left(u_{1}\right)\right. \\
& \left.-\frac{(\boldsymbol{H})(\boldsymbol{H}) \cdot \boldsymbol{e}}{|\boldsymbol{H}|^{2}+k_{\mathrm{c}}{ }^{2}} G^{2}\left(u_{2}\right)\right],
\end{aligned}
$$

where $G(u)=3(\operatorname{Sin} u-u \cos u) u^{-3}$. 
$\boldsymbol{e}$ is the unit polarization vector, $u_{1}$ and $u_{2}$ are as defined by Krebs [9] and $k_{\mathrm{c}}$ is the Bohm-Pine screening parameter $\left(k_{\mathrm{c}}=0.353\left(r_{0} / a_{0}\right)^{1 / 2} k_{\mathrm{F}} ; a_{0}, r_{0}\right.$ and $k_{\mathrm{F}}$ are Bohr's radius, the interelectronic distance, and the Fermi wave vector, respectively).

In the long wavelength limit $(q \rightarrow 0)$ the dynamical parameters $\left(\alpha_{1}, \alpha_{2}, \beta_{1}, \beta_{2}, K\right.$ and $\left.A^{\prime}\right)$ can be parameterised in terms of macroscopic elastic constants of fec lattices by the relations

$$
\begin{aligned}
\alpha_{1}+\beta_{1} & =-2 a C_{44}, \\
\alpha_{2}+\beta_{2} & =-\frac{1}{2}\left(C_{11}-C_{12}-C_{44}\right) a, \\
A^{\prime} & =\left(C_{12}-C_{44}\right) 2 a^{3} k_{\mathrm{c}}{ }^{2} .
\end{aligned}
$$

These equations, together with the three equations for the zone boundary of the transverse branches in the $[\zeta \zeta \zeta]$ and [ $\zeta 00]$ and of the longitudinal branch in [ $\zeta 00]$ directions form a sufficient set of equations to calculate the six unknown force constants of the model.

The frequency vs. wave vector dispersion curves along the highly symmetric directions, in which the lattice waves are either wholly longitudinal (L) or wholly transverse ( $\mathrm{T}$ ), are computed for $\mathrm{Cu}, \mathrm{Ag}$ and $\mathrm{Au}$ from the solution of the secular equation (1). The input data for finding the force constants of these metals are given in Table 1 and their calculated values in Table 2 . The elastic constants used are due to measurements of Overton and Gaffney [13] for $\mathrm{Cu}$ and Neighbours and Alers [14] for $\mathrm{Ag}$ and $\mathrm{Au}$.

In the relaxation time approximation, the phonon limited electrical resistivity is expressed by Ziman [15] as

$$
\varrho_{i}=\left(m / n z e^{2}\right) \tau_{\mathrm{e}^{-1}},
$$

where $e$ is the electronic charge, $m$ the bare electron mass, $n$ the number of ions per unit volume and $z$ the valency of the metal.

In the pseudopotential approximation and using the simplest trial function the relaxation time (for lattices with cubic symmetry) $\tau_{\mathrm{e}}^{-1}$ for electron scattering by lattice oscillations is expressed by Baym [16] as

$\tau_{\mathrm{e}}^{-1}=\frac{3}{16 \hbar m V_{\mathrm{F}^{2}} k_{\mathrm{F}^{4}}} \int_{\Omega}^{2} \int_{0}^{2 k_{\mathrm{F}}} S(\boldsymbol{q})|V(q)|^{2} q^{3} \mathrm{~d} q \mathrm{~d} \Omega$,

where $k_{\mathrm{F}}$ is the Fermi wave vector, $\Omega$ the solid angle in the wave vector space, $V(\boldsymbol{q})$ the pseudopotential form factor and $V_{F}$ the Fermi velocity. $S(\boldsymbol{q})$, the structure factor depending solely on the lattice dynamics of the material, is given by

$$
\begin{aligned}
S(\boldsymbol{q})= & \frac{\hbar^{2}}{M k_{\mathrm{B}} T} \\
& \cdot \sum_{j} \frac{\left(\boldsymbol{e}_{\boldsymbol{q} j} \cdot \boldsymbol{q}\right)^{2}}{\left(\exp \left\{\beta \omega_{\boldsymbol{q} j}\right\}-1\right)\left(1-\exp \left\{-\beta \omega_{\boldsymbol{q} j}\right\}\right)},
\end{aligned}
$$

where $M$ is the mass of an atom, $\omega_{\boldsymbol{q} j}$ the angular frequency of the phonon wave vector $\boldsymbol{q}$ with polarization index $j, \boldsymbol{e}_{\boldsymbol{q} j}$ the polarization vector and $\beta=\hbar / k_{\mathrm{B}} T$.

In deducing the expression (11), it has been assumed that the Fermi surface is spherical and the pseudopotential matrix element $\left\langle k^{\prime}|V| k\right\rangle$ is a function of $\boldsymbol{q}$ alone.

Here we report an investigation of the tempera-

\begin{tabular}{|c|c|c|c|c|c|c|c|}
\hline \multirow[t]{3}{*}{ Metals } & \multicolumn{3}{|c|}{$\begin{array}{l}\text { Elastic Constants } \\
\left(\times 10^{11} \mathrm{dyn}_{\mathrm{cm}^{-2}}\right)\end{array}$} & \multirow{3}{*}{$\begin{array}{l}\text { Lattice } \\
\text { param- } \\
\text { eter } \\
\lambda a(\AA)\end{array}$} & \multicolumn{3}{|c|}{$\begin{array}{l}\text { Phonon frequencies }(\mathrm{THz}) \\
\text { in the direction }\end{array}$} \\
\hline & \multirow[t]{2}{*}{$C_{11}$} & \multirow[t]{2}{*}{$C_{12}$} & \multirow[t]{2}{*}{$C_{44}$} & & {$[\zeta 00]$} & & {$[\zeta \zeta \zeta]$} \\
\hline & & & & & $\overline{v_{\mathrm{L}}}$ & $\nu_{\mathrm{T}}$ & $\nu_{\mathrm{T}^{\prime}}$ \\
\hline Copper & 16.839 & 12.142 & 7.539 & 3.6147 & 7.20 & 5.09 & 3.40 \\
\hline Silver & 12.399 & 9.367 & 4.612 & 4.080 & 4.95 & 3.43 & 2.25 \\
\hline Gold & 19.234 & 16.314 & 4.195 & 4.078 & 4.61 & 2.75 & 1.86 \\
\hline
\end{tabular}
ture dependence of the ideal electrical resistivity of

\begin{tabular}{|c|c|c|c|c|c|c|}
\hline Metal & $\alpha_{1}$ & $\alpha_{2}$ & $\beta_{1}$ & $\beta_{2}$ & $K$ & $A^{\prime}$ \\
\hline Copper & -26.9946 & 2.3656 & -0.2566 & 0.20275 & 0.0897 & 33.3719 \\
\hline Silver & -23.9394 & 0.4437 & 5.1224 & 1.1679 & 33.9174 & 43.9167 \\
\hline Gold & 25.687 & 12.4429 & -42.794 & -11.1431 & 1233.2842 & 111.8203 \\
\hline
\end{tabular}
noble metals using pseudopotentials for electron ion scattering. The pseudopotential form factors $V(\boldsymbol{q})$ are taken from the Moriarty [17, 18] and Borchi and de Gennaro [19] models. The choice of
Table 1. Input data to calculate atomic force constants.

Table 2. Output values of force constants in units of $10^{3} \mathrm{dyn}$ $\mathrm{cm}^{-1}$. 
these pseudopotential models is warranted by their success in the understanding of various metallic properties. The structure factor $S(\boldsymbol{q})$ is determined from Eq. (12) using our model for phonon frequencies and polarization vectors. The integration over $\boldsymbol{q}$ is performed numerically and the angular integration is done by using a modification of Houston's spherical six term integration procedure as elaborated by Betts et al. [20]. The results obtained from a six term approximation are reported. The six directions used for $q$ 's are [100], [110] and [111], [210], [211] and [221].

\section{Results and Discussion}

The calculated lattice waves dispersion curves along the three directions [ $\zeta 00]$, $[\zeta \zeta 0]$ and $[\zeta \zeta \zeta]$ for $\mathrm{Cu}, \mathrm{Ag}$ and $\mathrm{Au}$ are plotted in Figs. $1-3$ along with the measured values of Svensson et al. [21], Kamitakahara and Brockhouse [22] and Lynn et al. [23], respectively. The agreement between the theoretical and experimental curves is very good for $\mathrm{Cu}$, but not so in the case of $\mathrm{Ag}$ and $\mathrm{Au}$ for the

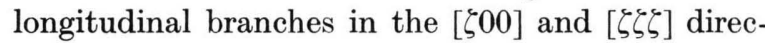
tions.

The calculated ideal electrical resistivities are plotted in Figs. 4-6 versus temperature. For comparison, we have also plotted the experimental values available from recent measurements. The most detailed observations of the electrical resistivity of noble metals have been performed by White and Woods [24] in the temperature range $200-300 \mathrm{~K}$.

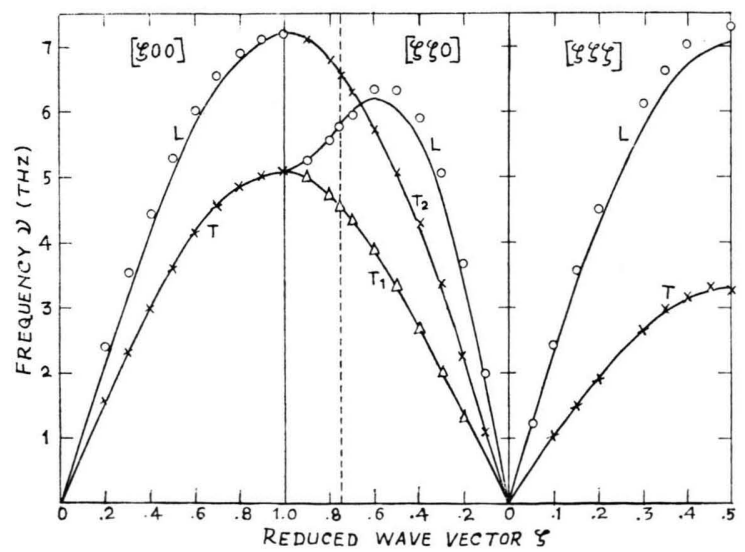

Fig. 1. Dispersion curves along the symmetry directions for copper. Full curves correspond to present calculations. The experimental points $O, \times$ and $\triangle$ are of Svensson et al. [21].

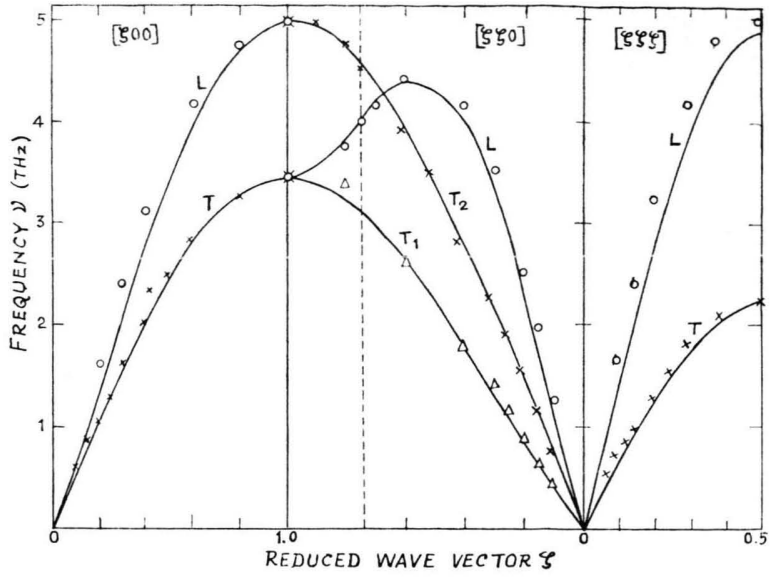

Fig. 2. Dispersion curves along the symmetry directions for silver. Full curves correspond to present calculations. The experimental points $O, \times$ and $\Delta$ are of Kamitakahara and Brockhouse [22].

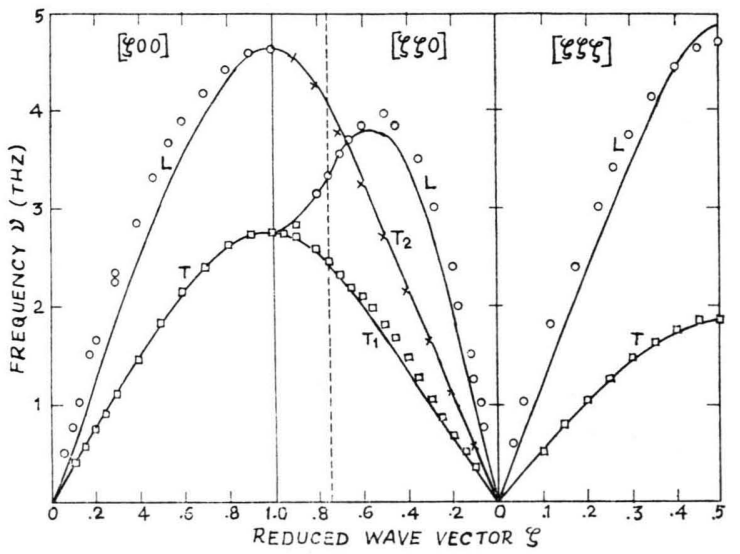

Fig. 3. Dispersion curves along the symmetry directions for gold. Full curves correspond to present calculations. Experimental points $O, \times$ and $\square$ are of Lynn et al. [23].

Their experimental values are in close agreement with the recent investigations of Moore et al. [25] for copper and of Matsumura and Laubitz [26] for silver in the temperature range $80-350 \mathrm{~K}$.

As is seen from Figs. 4-6, the general behaviour of the computed curves is very similar to that of the experimental ones. Thus the present study indicates that the temperature dependence of the electrical resistivity predicted by Ziman-Baym's theory and employing our model for the phonon spectrum is reasonably consistent with experiment. The results further show that the electrical resistivity of metals is very sensitive to the detailed variation of the pseudopotential form factors. 


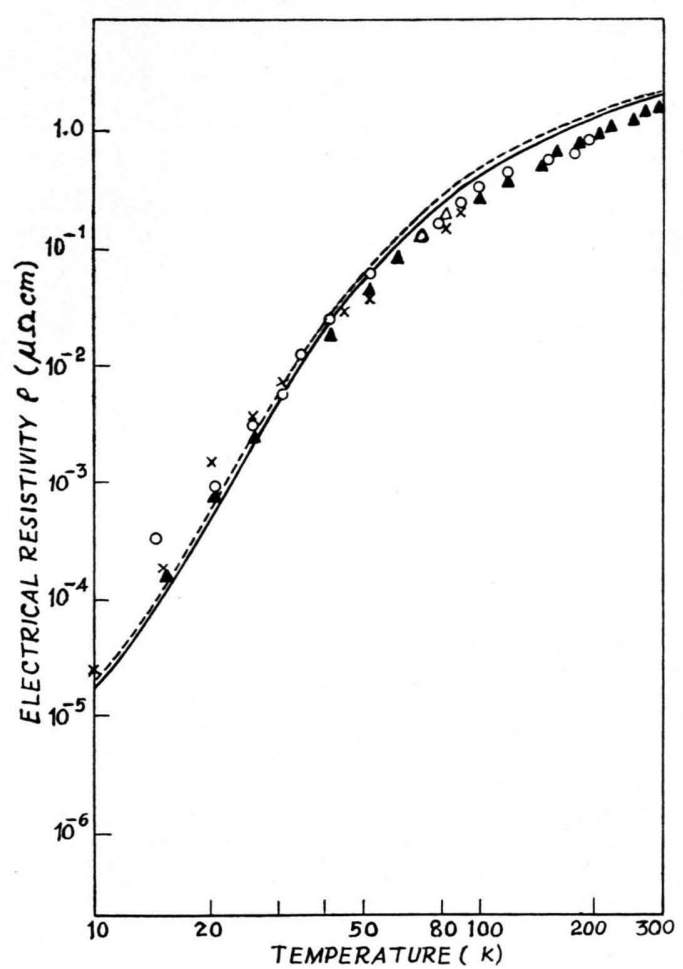

Fig. 4.

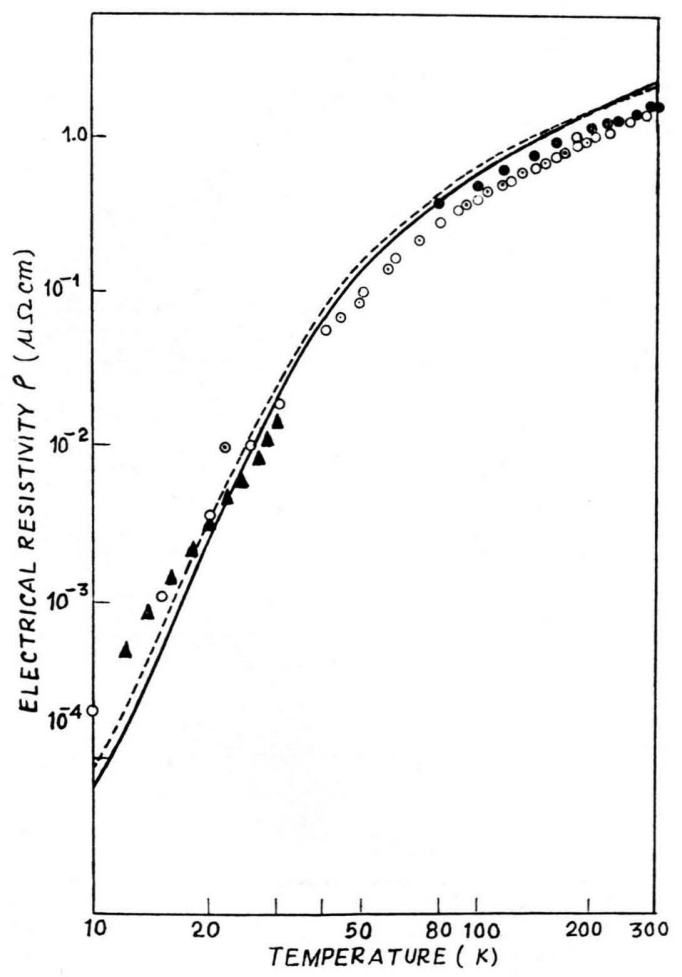

Fig. 5.
Fig. 4. Temperature variation of electrical resistivity of copper. The broken curves are due to the Moriarty and the full curves due to the Borchi and de Gennaro models. The experimental points $(\circ \circ \circ \circ)$ are due to White [29], $(\boldsymbol{\Delta} \Delta \mathbf{\Delta} \boldsymbol{\Delta})$ due to White and Woods [24] and $(x \times x \times)$ due to Berman and MacDonald [30].

The discrepancies between theory and experiment observed in the present study are not unexpected and may be attributed to the neglect of ion core-ion core angular interactions, ion-core-conduction electrons interactions, the assumption of short range interactions $[27,28]$ and the neglect of the flexibility of d-orbitals in the present calculations. The neglect

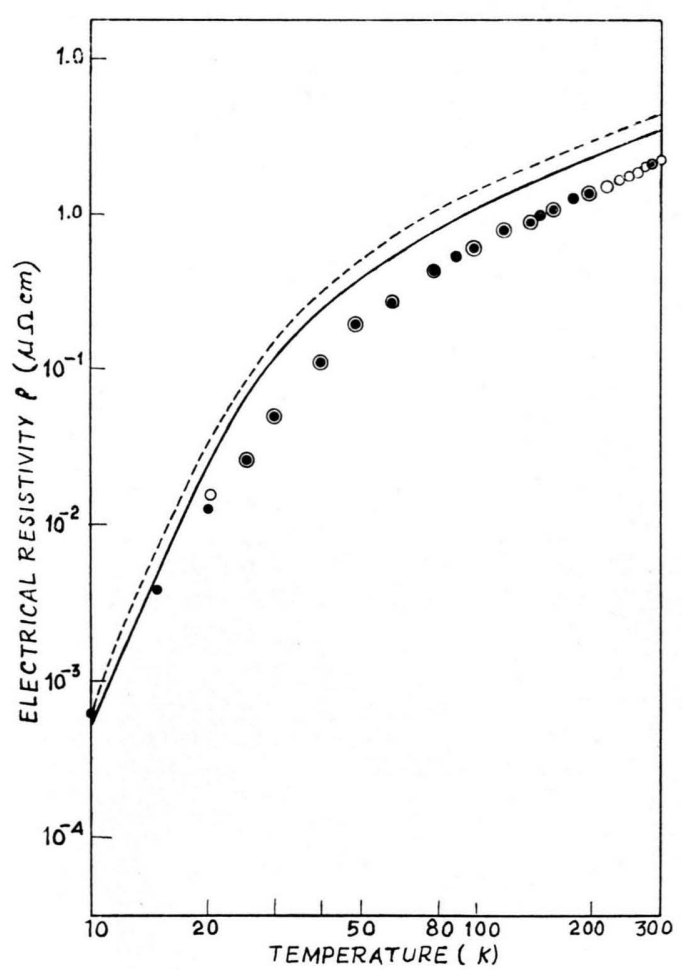

Fig. 6. Temperature variation of electrical resistivity of gold. The full curve is due to the Moriarty and the broken curve due to the Borchi and de Gennaro models. The experimental points $(\bullet \bullet \bullet \bullet)$ are the measurements of White and Woods [24] and ( $\circ \circ \circ \circ)$ of Cook and VanderMeer [33].

Fig. 5. Temperature variation of electrical resistivity of silver. The broken curves are due to the Moriarty and the full curves due to the Borchi and de Gennaro models. The experimental points $(0 \circ \circ \circ)$ are the measurements of

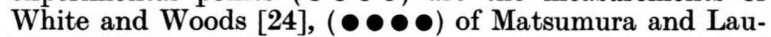
bitz [26], ( $\mathbf{\Delta} \mathbf{\Delta} \mathbf{\Delta})$ of Fenton et al. [31] and $(\odot \odot \odot \odot)$ of Dugdale and Basinski [32]. 
of anharmonic contributions such as the Debye Waller factor and various multiphonon processes in the lattice may, however, also contribute to the discrepancies in the electrical resistivity. At higher temperatures multiphonon processes become important and the one phonon approximation, on which formula (12) is based, become less valid.

[1] R. Cavalheiro and M. M. Shukla, Nuovo Cim. 26 B, 220 (1975).

[2] C. M. Coel, B. P. Pande, and B. Dayal, Phys. Stat. Sol. (b) 63, 625 (1974).

[3] V. P. Singh and M. P. Hemkar, Phys. Lett. 54 A, 24 (1975).

[4] M. M. Shukla and H. Closs, J. Phys. F 3, L1 (1973).

[5] S. Jogi, J. Phys. F4, 11 (1974).

[6] J. de Launay, J. Chem. Phys. 21, 1975 (1953).

[7] A. B. Bhatia, Phys. Rev. 97, 363 (1955).

[8] P. K. Sharma and S. K. Joshi, J. Chem. Phys. 39, 2633 (1963).

[9] K. Krebs, Phys. Rev. 138 A, 143 (1965); Phys. Lett. 10, 12 (1964).

[10] L. Cheveau, Phys. Rev. 169, 496 (1968).

[11] B. L. Fielek, J. Phys. F5, 17 (1975).

[12] B. P. Sing, L. P. Pathak, and M. P. Hemkar, J. Phys. F11 (1978).

[13] W. C. Overton and J. Gaffney, Phys. Rev. 98, 969 (1955).

[14] J. R. Neighbours and G. A. Alers, Phys. Rev. 111, 707 (1958).

[15] J. M. Ziman, Electrons and Phonons, Oxford University Press, New York 1960.

[16] G. Baym, Phys. Rev. 135 A, 1691 (1964).

[17] J. A. Moriarty, Phys. Rev. B 1, 1363 (1970).

[18] J. A. Moriarty, Phys. Rev. B 6, 1239 (1972).

[19] G. Borchi and S. de Gennaro, Phys. Lett. 32 A, 301 (1970).

\section{Acknowledgements}

The authors are very grateful to Professor Vachaspati for providing adequate facilities in the department and to Professor Bipin K. Agarwal for taking keen interest in this work. One of us (B.P.S.) thankfully acknowledges the "Teacher Fellowship" awarded by U.G.C., New Delhi.

[20] D. D. Betts, A. B. Bhatia, and M. Wyman, Phys. Rev. 104, 37 (1956).

[21] E. C. Svensson, B. N. Brockhouse, and J. M. Rowe, Phys. Rev. 155, 619 (1967).

[22] W. A. Kamitakahara and B. N. Brockhouse, Phys. Lett. 29 A, 639 (1969).

[23] J. W. Lynn, H. G. Smith, and R. M. Nicklow, Phys. Rev. B 8, 3493 (1973).

[24] G. K. White and S. B. Woods, Phil. Trans. Roy. Soc. A 251, 273 (1959).

[25] J. P. Moore, D. L. McEloy, and R. S. Girras, Canad. J. Phys. 45, 3849 (1967).

[26] T. Matsumura and M. J. Laubitz, Canad. J. Phys.48, 1499 (1970).

[27] B. N. Brockhouse, T. Arase, G. Caglioti, K. R. Rao, and A. D. B. Woods, Phys. Rev. 128, 1099 (1962).

[28] A. D. B. Woods, B. N. Brockhouse, R. H. March, A. J. Stewart, and R. Bowers, Phys. Rev. 128, 1112 (1962).

[29] G. K. White, Aust. J. Phys. 6, 397 (1953).

[30] R. Berman and D. K. C. MacDonald, Proc. Roy. Soc. London A 211, 122 (1952).

[31] E. W. Fenton, J. S. Rogers, and S. B. Woods, Canad. J. Phys. 41, 2066 (1963).

[32] J. S. Dugdale and Z. S. Basinski (Private communication).

[33] J. G. Cook and M.P. VanderMeer, Canad. J. Phys. 48, 224 (1970). 